\title{
Antinociceptive tolerance to NSAIDs in the agranular insular cortex is mediated by opioid mechanism
}

This article was published in the following Dove Press journal:

Journal of Pain Research

6 July 2017

Number of times this article has been viewed

\author{
Natia Pirkulashvili' \\ Nana Tsiklauri' \\ Marina Nebieridze 2 \\ Merab GTsagareli' \\ 'Laboratory of Pain and Analgesia, \\ ${ }^{2}$ Laboratory of Brain Metabolism, \\ Beritashvili Center for Experimental \\ Biomedicine, Tbilisi, Georgia
}

\begin{abstract}
Several lines of investigations have shown that in some brain areas, in particular, in the midbrain periaqueductal gray matter, rostral ventromedial medulla, central nucleus of amygdala, nucleus raphe magnus, and dorsal hippocampus, microinjections of nonsteroidal anti-inflammatory drugs (NSAIDs) induce antinociception with distinct development of tolerance. The agranular insular cortex (AIC) is a small region of the cerebral cortex located on the lateral area of the rat's cerebral hemisphere that is involved in the perception and response to pain. In the present study, we investigated the development of tolerance to the analgesic effects of NSAIDs diclofenac, ketorolac, and xefocam microinjected into the AIC in rats. Male Wistar rats receiving NSAIDs into the AIC were tested for antinociception by tail-flick and hot plate tests. Treatment with each NSAID significantly enhanced the tail-flick and hot plate latencies on the first day, followed by a progressive decrease in the analgesic effect over a 4-day period, ie, they developed tolerance. Pretreatment with an opioid antagonist naloxone completely prevented, and posttreatment naloxone abolished, the analgesic effects of the three NSAIDs in both behavioral assays. These findings support the notion that the development of tolerance to the antinociceptive effects of NSAIDs is mediated via an endogenous opioid system possibly involving descending pain modulatory systems.
\end{abstract}

Keywords: antinociception, endogenous opioids, descending modulation, nociception, nonopioid tolerance

\section{Introduction}

A growing body of literature suggests that the brain region that is a part of the pain processing network, the insula, is both anatomically and functionally well suited to serve a primary and fundamental role in pain processing. By quantitative perfusion neuroimaging to investigate slowly varying neural states highly relevant to a complex phenomenon, such as pain, Segerdahl et al $^{1}$ identified the dorsal posterior insula as subserving a fundamental role in pain and as the likely human homologue of the nociceptive region identified from animal studies. Especially with regard to pain experience, the insular cortex has been presumed to participate in both sensory-discriminative and affective-motivational aspects of pain. ${ }^{2}$

The agranular insular cortex (AIC) is found in other mammals including cats, monkeys, and primates, including humans. In primates, the divisions of the insular cortex are the same as in rats, and the AIC occupies an area immediately noticeable, that is, dorsal to the primary olfactory cortex. The rat's AIC is a small region of the cerebral cortex located on the lateral area of the cerebral hemisphere that is involved
Correspondence: Merab G Tsagarel Beritashvili Center for Experimental Biomedicine, 14 Gotua Street, 0160 Tbilisi, Georgia

Tel +99532 237 II 49

Fax +99532 2373410

Email m.tsagareli@biomedicine.org.ge 
in the perception and response to pain. ${ }^{3}$ Direct injections of morphine into the AIC increase dopamine and GABA levels, resulting in behavioral antinociception. ${ }^{4}$ The major connections of the AIC are with areas that have established roles in behavior responses to nociceptive stimuli. The AIC projections to other cortical areas and subcortical sites such as the amygdala are likely to participate in the sensorimotor integration of nociceptive processing, while the hypothalamus and brainstem projections are most likely to contribute to descending pain inhibitory control. ${ }^{3}$

Nonsteroidal anti-inflammatory drugs (NSAIDs) are the most widely used analgesics in the treatment of mild pain. For the last few years, however, it was established that repeated administration of NSAIDs systemically or in the midbrain periaqueductal gray matter (PAG) induced tolerance to these drugs, similar to opioid analgesics, and cross-tolerance to morphine. ${ }^{5-11}$

We have recently shown that tolerance develops to analgesic effects of the commonly used NSAIDs (metamizol, diclofenac, ketorolac, and xefocam) given intraperitoneally in juvenile and adult rats in models of acute and chronic pain (the formalin test). ${ }^{12,25}$ We have also revealed that repeated microinjections of these nonopioids into the dorsal hippocampus (DH), the nucleus raphe magnus (NRM), and the central nucleus of amygdala (CeA) induce antinociception and the effects of tolerance and cross-tolerance to morphine. .,, $13-15^{-15}$ These findings strongly support the suggestion of endogenous opioids involvement in NSAIDs antinociception and tolerance in the descending pain-control system. ${ }^{8,16}$

In the present study, we hypothesized that the analgesic effects of the three NSAIDs, diclofenac, ketorolac, and xefocam microinjected into the AIC would exhibit antinociceptive tolerance mediated via endogenous opioids.

\section{Materials and methods Animals}

The research was carried out on adult male Wistar rats weighing 200-250 g, bred at the Beritashvili Exp. BMC. The animals were kept under standard housing conditions $\left(22^{\circ} \mathrm{C} \pm 2{ }^{\circ} \mathrm{C}, 65 \%\right.$ humidity, and light from 7:00 a.m. to 8:00 p.m.) and kept on a standard dry diet with water freely available. Every effort was made to minimize both the number of animals used and their suffering. Six rats were used for each experimental and control group. The local Bioethic Committee of the Beritashvili Center for Experimental Biomedicine approved the experimental protocols, and we adhered to the Guidelines of the International Association for the Study of Pain regarding investigations of experimental pain in conscious animals. ${ }^{17}$

\section{Surgical procedures}

Under anesthesia with intramuscular administration of ketamine (100 mg/kg, "KharkovPharm", Ukraine), a 12-mm-long stainless steel guide cannula (Small Parts, Inc., Logansport, IN, USA) was stereotaxically implanted bilaterally into the rostral part of AIC, (AP: 2.70; L: \pm 4.4 ; $\mathrm{H}: 5.8)$ according to the coordinates in the atlas of Paxinos and Watson, ${ }^{18}$ siting the tip $2 \mathrm{~mm}$ above the AIC. The guides were anchored to the cranium using dental cement. The guide cannula was plugged with a stainless steel stylet. Thereafter, the animals were handled every day for 3-4 days for 15-20 minutes to get familiar with the testing protocol and experimental environment. During this time, the stylet was removed and a 14-mm long stainless steel microinjection cannula was inserted into the guide cannula to reach the AIC, but no drug was injected. Five days after surgery, the microinjection cannula, attached to Hamilton syringe (Hamilton, Inc., McLean, VA, USA), was joined to the guide cannula, and the drug was introduced through it while the rat was gently restrained.

\section{Drugs}

Diclofenac (diclofenac sodium, $75 \mu \mathrm{g} / 0.5 \mu \mathrm{L}$, Hemofarm, Vršac, Serbia), ketorolac (ketorolac tromethamine, $90 \mu \mathrm{g} / 0.5 \mu \mathrm{L}$, Grindex, Latvia), or xefocam (lornoxicam, $12 \mu \mathrm{g} / 0.5 \mu \mathrm{L}$, Nycomed, Zürich, Austria) were injected through the microinjection cannula as used in previous works. ${ }^{13,14}$ The guide cannula was then plugged with a stainless steel stylet. Isotonic saline was injected in the same volume $(0.5 \mu \mathrm{L}$, GalichPharm, Ukraine) and manner in a separate group of rats, treated as controls. In the second set of experiments, a nonselective opioid receptor antagonist naloxone $(0.2 \mu \mathrm{g} / 0.5 \mu \mathrm{L}$, Polfa S.A., Poland) was injected through the microinjection cannula. ${ }^{13,14}$ Solutions were microinjected in about $10-12$ seconds.

\section{Behavioral testing}

Twenty minutes after microinjection of NSAIDs or saline, ie, 10 minutes before the peak of the drugs' effect is normally reached, rats were tested for antinociception using the tailflick (TF) and hot plate (HP) tests. For the TF test, the distal part of the tail was stimulated with a light beam and the latency measured until the tail was reflexively flicked away from the beam (IITC \#33, IITC Life Science, Inc., Woodland Hills, CA, USA). For the HP test, the rat was placed on a $55^{\circ} \mathrm{C}$ hot plate and the latency to the first hindpaw lick or time to first jump was measured (IITC \#39). The cut off time was $20 \mathrm{~s}$ for both TF and HP latencies. Each animal was tested with 
both TF and HP tests in the same session. A similar procedure was followed for the repeated microinjection of diclofenac, ketorolac, xefocam, or saline for four consecutive days.

In the second set of experiments, pretreatment of rats with naloxone in the AIC was followed by TF and HP tests 10 minutes after they were treated with NSAIDs in the same dose as in the first set of experiments and were then retested again.

In the third set of experiments, posttreatment of rats with naloxone in the AIC was followed by TF and HP tests. For this purpose, 10 minutes prior to naloxone microinjections, rats were pretreated with NSAIDs in the same dose as in the first and second set of experiments and were then retested again. Different animal groups were used for the first, second, and third sets of experiments. The number of rats in each group was six.

\section{Histology}

At the end of each set of experiments, the microinjection sites were marked with $2 \mu \mathrm{L}$ of saturated solution of Pontamine Sky Blue (Sigma-Aldrich, St. Louis, MO, USA) and the animal was euthanized with pentobarbital. After fixation by immersion in $10 \%$ formalin, the brain was sectioned and counterstained with Cresyl Violet. The microinjection sites were histologically verified and plotted according to Paxinos and Watson ${ }^{18}$ stereotaxic atlas coordinates. Representative microinjection sites are shown in the Figure 1. However, we are aware that our histology needs more evidence for accuracy of the location of the injection sites, which limits the interpretation of the obtained data.

\section{Statistical analysis}

All mean control and experimental groups' values are presented as mean \pm standard error of mean. One-way analysis of variance (ANOVA) or repeated-measures ANOVA with post hoc Tukey-Kramer or Dunnett's multiple comparison tests were used for statistical evaluation of comparisons between treated and saline groups and between treated and naloxone groups, respectively. The Kolmogorov-Smirnov test was applied to verify normality. The statistical software utilized was InStat 3.05 (GraphPad Software, San Diego, CA, USA). Differences between means of saline control and treated groups and between naloxone and treated groups of rats were acknowledged as statistically significant if $P<0.05$.

\section{Results}

\section{Tolerance to antinociceptive effects of NSAIDs}

In the first set of experiments, we found that microinjection of NSAIDs into the AIC produced antinociception, as revealed by a latency increase in the TF and HP compared to the baseline control of intact rats and a control group with saline microinjected into the same site. The repeated-measures ANOVA revealed that the TF latency significantly increased for clodifen $[\mathrm{F}(9,20)=56.229, P<0.0001]$, ketorolac
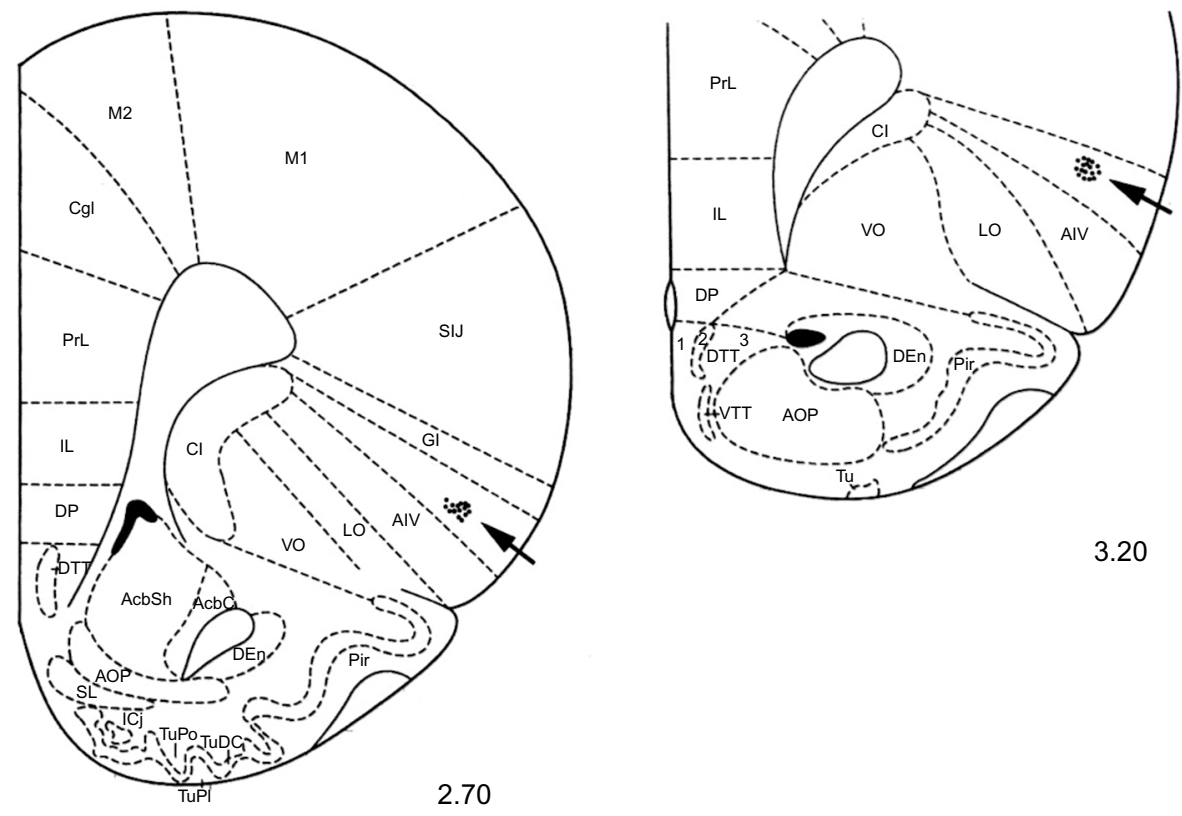

3.20

Figure I Serial coronal sections of the rat brain showing placement of microinjections in the AIC (black arrows).

Notes: The number below each section represents millimeters relative to bregma. Adapted from the Stereotaxic atlas in Paxinos G, Watson C. The Rat Brain in Stereotaxic Coordinates. Compact third edition. San Diego, CA: Academic Press; 1997. Copyright @ 1997 by George Paxinos and Charles Watson. ${ }^{18}$

Abbreviation: AIC, agranular insular cortex. 
$[\mathrm{F}(9,20)=30.398, P<0.0001]$, and xefocam $[\mathrm{F}(9,20)=53.058$, $P<0.0001]$, respectively, but not for saline group $[\mathrm{F}(9$, $20)=1.941, P=0.1428$, not significant $]$. On the first experimental day, the differences between NSAIDs-treated groups and the intact control group were significant for diclofenac $(t=18.549, P<0.001)$, for ketorolac $(t=12.024, P<0.001)$, and for xefocam $(t=17.696, P<0.001)$, respectively (Figure $2 \mathrm{~A})$. The TF latency differences between NSAIDs-treated groups and the saline control group by Dunnett's test were significant on the first experimental day for diclofenac ( $t=4.940$, $P<0.01)$, ketorolac $(t=2.541, P<0.05)$, and xefocam $(t=5.733$, $P<0.01$ ), respectively.

We found similar significant differences of enhancement in the HP latencies compared to the intact group for diclofenac $[\mathrm{F}(9,20)=51.749, P<0.0001]$, for ketorolac $[\mathrm{F}(9,20)=43.359$,
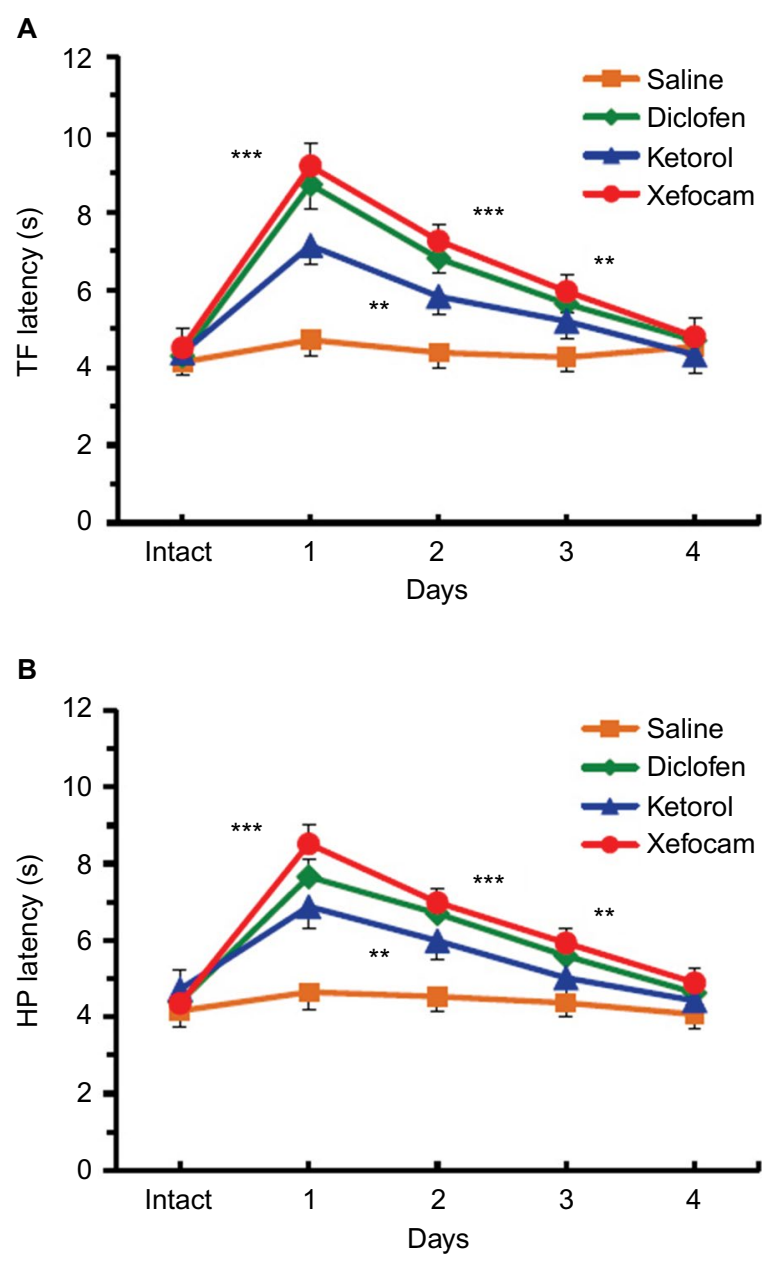

Figure 2 Microinjections of NSAIDs into the AIC for 4 consecutive days. Notes: (A) A progressive decrease in TF latency as compared to intact control group; (B) a progressive decrease in HP latency to intact control group. Statistical analysis was performed by repeated-measure ANOVA with post hoc TukeyKramer's multiple comparisons test; $n=6$ per test group; ${ }^{* *} P<0.01$, $* * * P<0.001$. Abbreviations: AIC, agranular insular cortex; ANOVA, analysis of variance; HP, hot plate; NSAIDs, nonsteroidal anti-inflammatory drugs; TF, tail-flick.
$P<0.0001]$, and for xefocam $[\mathrm{F}(9,20)=38.551, P<0.0001]$, respectively, but not for saline control $[\mathrm{F}(9,20)=2.681$, $P=0.0613$, not significant $]$. Here also on the first experimental day, the differences between NSAIDs-treated groups and the intact control group were significant for clodifen $(t=16.989$, $P<0.001)$, for ketorolac $(t=14.209, P<0.001)$, and for xefocam $(t=15.488, P<0.001)$, respectively (Figure $2 \mathrm{~B})$. The HP latency differences between NSAIDs-treated groups and the saline control group by Dunnett's test were significant in the first experimental day for diclofenac $(t=6.938, P<0.01)$, for ketorolac $(t=5.012, P<0.01)$, and for xefocam $(t=7.580$, $P<0.01)$.

Subsequent NSAIDs microinjections into the AIC caused gradually less antinociception, so by day 4 there was no effect, similar to saline microinjections for both the TF and the HP tests, i.e. induced tolerance (Figure 2). On the second experimental day, the TF latency differences between NSAIDs-treated groups and the saline control group by Dunnett's test were significant for xefocam $(t=4.065, P<0.01)$ and diclofenac $(t=3.090, P<0.05)$, but not for ketorolac $(t=0.9749$, $P>0.05$, not significant). On the third and fourth experimental days, there were no significant differences between NSAIDstreated groups and the saline control.

Concerning the HP test, on the second experimental day, the latency differences between NSAIDs-treated groups and the saline group by Dunnett's test were significant for diclofenac $(t=4.534, P<0.01)$, xefocam $(t=4.576, P<0.01)$, and ketorolac $(t=2.899, P<0.05)$, respectively. On the third day there, were significant differences for xefocam $(t=3.345$, $P<0.01)$ and diclofenac $(t=3.002, P<0.05)$, but not for ketorolac $(t=1.598, P>0.05$, not significant), while on the fourth day there were no significant differences for any of the NSAIDs.

\section{Pretreatment with naloxone prevents NSAIDs-induced antinociception}

In the second set of experiments, we tested if pretreatment with a nonselective opioid receptor antagonist naloxone prevents antinociception induced by NSAID microinjected into the AIC. Pretreatment with naloxone completely prevented the analgesic effects of diclofenac, ketorolac, and xefocam in the TF test. The ANOVA did not reveal significant differences between naloxone-injected and NSAIDs-injected groups for diclofenac $[\mathrm{F}(2,15)=0.6083, P=0.2552$, not significant $]$, for ketorolac $[\mathrm{F}(2,15)=0.8998, P=0.4275$, not significant $]$, and for xefocam $[\mathrm{F}(2,15)=3.078, P=0.0758$, not significant $]$, respectively, and hence the TF latencies of the saline, naloxone, and NSAIDs groups were not significantly different when compared with the post hoc test $(P>0.05)$ (Figure $3 \mathrm{~A}, \mathrm{C}$ and $\mathrm{E}$ ). 

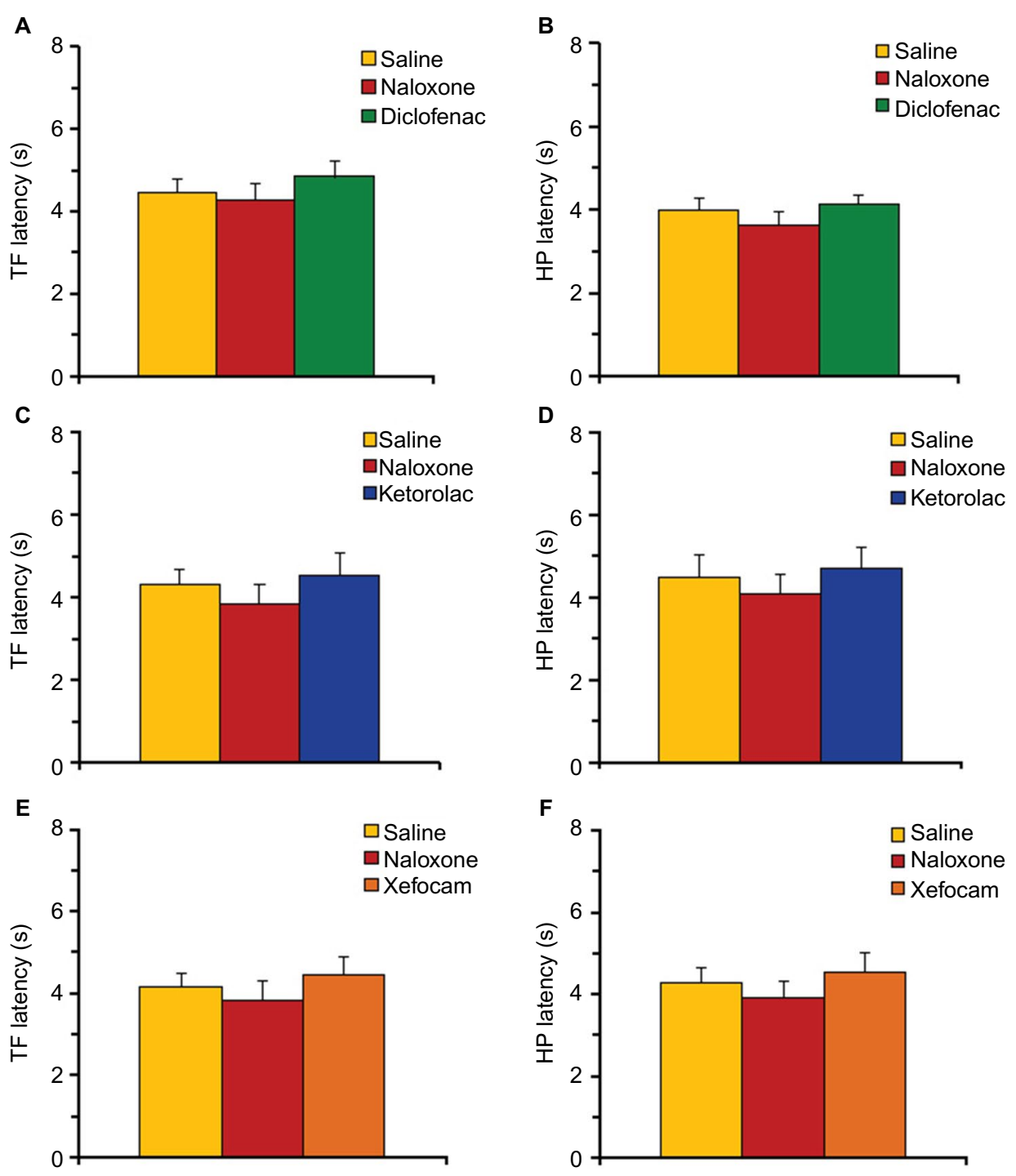

Figure 3 Pretreatment with naloxone $(0.2 \mu g / 0.5 \mu \mathrm{L})$ before microinjections of NSAIDs into the AIC.

Notes: (A, C and E) Naloxone prevents NSAID-induced antinociception in TF latency for diclofenac (A), ketorolac (C), and xefocam (E), respectively. (B, D and F) Naloxone prevents NSAID-induced antinociception in HP latency for diclofenac (B), ketorolac (D), and xefocam (F), respectively. Statistical analysis was performed by oneway ANOVA with post hoc Tukey-Kramer's multiple comparisons test; $n=6$ per test group.

Abbreviations: AIC, agranular insular cortex; ANOVA, analysis of variance HP, hot plate; NSAIDs, nonsteroidal anti-inflammatory drugs; TF, tail-flick.

Similar results were observed in the HP test for diclofenac $[\mathrm{F}(2,15)=0.8492, P=0.4473$, not significant $]$, for ketorolac $[\mathrm{F}(2,15)=0.3815, P=0.6893$, not significant $]$, and for xefocam $[\mathrm{F}(2,15)=0.6152, P=0.5536$, not significant $]$, respectively, and hence the HP latencies of the saline, naloxone, and NSAIDs groups were not significantly different when compared with the post hoc test $(P>0.05)$ (Figure $3 \mathrm{~B}, \mathrm{D}$ and $\mathrm{F}$ ).

\section{Posttreatment with naloxone abolishes NSAIDs-induced antinociception}

In the third set of experiments, we tested if posttreatment with naloxone abolishes antinociception induced by NSAID microinjected into the AIC. We found that posttreatment with naloxone completely abolished the analgesic effects of diclofenac, ketorolac and xefocam in the TF test. The ANOVA revealed significant differences in the TF latencies between saline, NSAIDs, and naloxone groups for diclofenac $[\mathrm{F}(2,15)=87.881, P<0.0001]$, for ketorolac $[\mathrm{F}(2,15)=89.175$, $P<0.0001]$, and for xefocam $[\mathrm{F}(2,15)=93.530, P<0.0001]$, respectively. Naloxone completely abolished antinociceptive effects of diclofenac $(t=15.914, P<0.001)$, ketorolac $(t=17.417, P<0.001)$, and xefocam $(t=15.157, P<0.001)$, respectively (Figure $4 \mathrm{~A}, \mathrm{C}$ and $\mathrm{E}$ ).

Similar results we obtained in HP test. Here also, the ANOVA revealed significant differences in the HP latencies between saline, NSAIDs, and naloxone groups for diclofenac $[\mathrm{F}(2,15)=86.529, P<0.0001]$, ketorolac $[\mathrm{F}(2,15)=39.425$, $P<0.0001]$, and xefocam $[\mathrm{F}(2,15)=114.22, P<0.0001]$, 

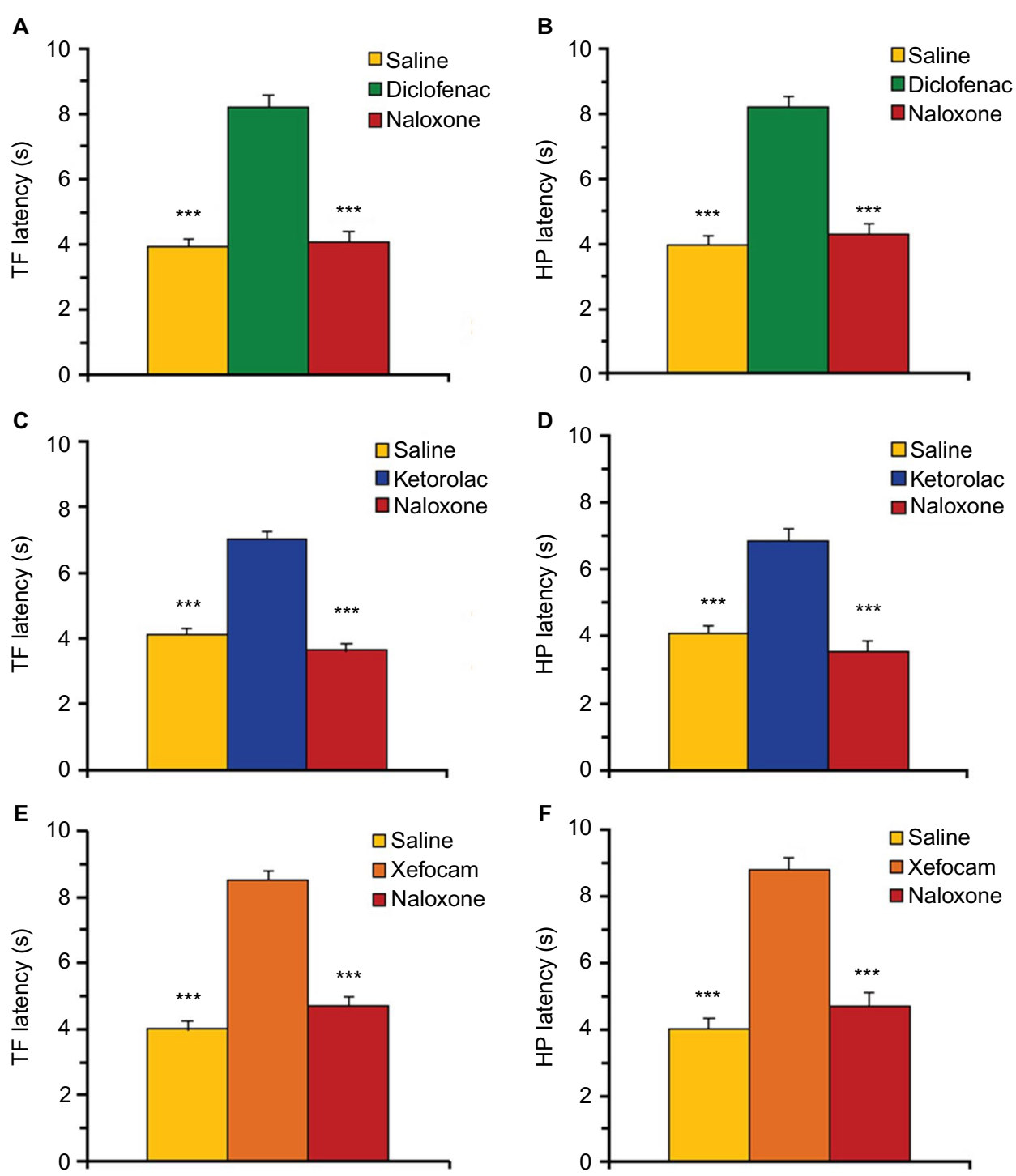

Figure 4 Posttreatment with naloxone $(0.2 \mu \mathrm{g} / 0.5 \mu \mathrm{L})$ after microinjections of NSAIDs into the AIC.

Notes: (A, C and E) Naloxone abolishes NSAID-induced antinociception in TF latency for diclofenac (A), ketorolac (C), and xefocam (D), respectively. (B, D and F) Naloxone abolishes NSAID-induced antinociception in HP latency for diclofenac (B), ketorolac (D), and xefocam (F), respectively. Each NSAID-injected group shows significant difference vs saline control and vs naloxone posttreated groups in both tests. Statistical analysis was performed by one-way ANOVA with post hoc TukeyKramer's multiple comparisons test; $\mathrm{n}=6$ per test group. $* * * \mathrm{p}<0.001$.

Abbreviations: AIC, agranular insular cortex; ANOVA, analysis of variance HP, hot plate; NSAIDs, nonsteroidal anti-inflammatory drugs; TF, tail-flick.

respectively. Opioid antagonist naloxone completely abolished analgesic effects of diclofenac $(t=15.503, P<0.001)$, ketorolac $(t=11.679, P<0.001)$, and xefocam $(t=17.022$, $P<0.001$ ), respectively (Figure 4B, D and F).

\section{Discussion}

The present data have demonstrated that microinjections of commonly used NSAIDs, diclofenac, ketorolac and xefocam, into the AIC induce antinociception. These results are similar to the findings of previous investigations in an acute pain model with TF and HP tests, and in which metamizol, xefocam, ketorolac or lysine-acetylsalicylate were given systemically or microinjected into the PAG, the CeA and the NRM. ${ }^{5,6,8,11,12,14,15,19,20}$ In another study, responses of spinal dorsal horn wide-dynamic range neurons to mechanical noxious stimulation in hindpaw of rats were strongly inhibited by intravenous metamizol. ${ }^{21}$

Moreover, repeated microinjections of these NSAIDs into the AIC over a period of 4 days resulted in development of tolerance due to a progressive decrease in antinociceptive effectiveness, reminiscent of that usually induced by opiates. ${ }^{5,7,8,12,22}$ These findings confirm our previous results in which development of tolerance was observed to the analgesic effects of diclofenac, ketorolac and xefocam microinjected 
into the DH of rats. After administration of each NSAID, a progressive decrease in TF and HP latency (ie, tolerance) was noticed over the 4-day period. ${ }^{13,20}$

According to the recently established concept, the mechanism producing tolerance to NSAIDs can be due to involvement of endogenous opioid peptides..$^{8,16,23,24}$ In this study, we have clearly shown that pre- and posttreatment of a nonselective opioid receptor antagonist naloxone significantly diminishes NSAIDs-induced antinociception. These findings confirm our previous evidence where NSAIDs antinociception in the DH was reduced by pretreatment as well as posttreatment with naloxone. ${ }^{13,20}$ We have just recently showed that systemic pretreatment with naloxone completely prevented the analgesic effects of intraperitoneally injected NSAIDs, in thermal paw withdrawal and mechanical paw withdrawal tests in the formalin model of pain. ${ }^{25}$

All presented data confirm the other results that antinociception induced by systemic metamizol involves endogenous opioids that can be blocked by naloxone at the levels of the PAG, the NRM and the spinal dorsal horn, as well as findings that endogenous opioids are involved in the potentiation of analgesia observed with a combination of morphine plus dipyrone. ${ }^{26,27}$ These data support a role for endogenous opioidergic descending pain-control circuits. The latter consists of the brainstem pain modulatory PAGrostral ventromedial medulla axis underscoring the strong convergence of antinociceptive mechanisms for nonopioid and opioid analgesics. ${ }^{6,16,23,24,28}$

\section{Conclusion}

Overall, we showed for the first time that microinjections of diclofenac, ketorolac, and xefocam, commonly used NSAID analgesics, into the AIC, induce antinociception in rats. Repeated administration leads to tolerance development to these drugs. The present data support the notion that the development of tolerance to the antinociceptive effects of NSAIDs is mediated via an endogenous opioid system, possibly involving descending pain modulatory systems. Nevertheless, future experimental trials with clear histology of the location of the injections should be cognizant of this antinociceptive tolerance phenomenon in the AIC in assessing treatment responses as well as probing underlying mechanisms.

\section{Acknowledgment}

We would like to thank Ms N Skhirtladze for her English editing of the manuscript.

\section{Disclosure}

The authors report no conflicts of interest in this work.

\section{References}

1. Segerdahl AR, Mezue M, Okell TW, John T Farrar, Tracey I. The dorsal posterior insula subserves a fundamental role in human pain. Nature Neurosci. 2015;18(4):499-503.

2. $\mathrm{Lu} \mathrm{C}$, Yang $\mathrm{T}$, Zhao $\mathrm{H}$ et al. Insular cortex is critical for the perception, modulation, and chronification of pain. Neurosci Bull. 2016;32(2): 191-201.

3. Jasmin L, Ohara PT. The rostral agranular insular cortex. In: Basbaum AI, Bushnell MC, editors. Science of Pain. Oxford: Elsevier; 2009: 717-722.

4. Jasmin L, Rabkin SD, Granato A, Boudah A, Ohara PT. Analgesia and hyperalgesia from GABA-mediated modulation of the cerebral cortex. Nature. 2003;424(6946):316-320.

5. Pernia-Andrade AJ, Tortorici V, Vanegas H. Induction of opioid tolerance by lysine-acetylsalicylate in rats. Pain. 2004;111(1-2):191-200.

6. Tortorici V, Aponte Y, Acevedo H, Nogueira L, Vanegas H. Tolerance to non-opioid analgesics in PAG involves unresponsiveness of medullary pain-modulating neurons in male rats. Eur J Neurosci. 2009;29(6):1188-1196.

7. Tortorici V, Nogueira L, Aponte Y, Vanegas H. Involvement of cholecystokinin in the opioid tolerance induced by dipyrone (metamizol) microinjections into the periaqueductal gray matter of rats. Pain. 2004;112(1-2):113-120.

8. Tsagareli MG, Tsiklauri N. Behavior Study of 'Non-opioid Tolerance'. New York, NY: Nova Biomedical; 2012.

9. Tsagareli MG, Tsiklauri N, Nozadze I, Gurtskaia G. Tolerance effects of NSAIDs microinjected into central amygdala, periaqueductal grey, and nucleus raphe: possible cellular mechanism. Neural Regen Res. 2012;7(13):1029-1039.

10. Vanegas H, Tortorici V. Opioidergic effects of non-opioid analgesics on the central nervous system. Cell Mol Neurobiol. 2002;22(5-6):655-661.

11. Vanegas $H$, Tortorici V. The periaqueductal gray as critical site for antinociception and tolerance induced by non-steroidal anti-inflammatory drugs. In: Maione S, Di Marzo V, editors. Neurotransmission in the Antinociceptive Descending Pathway. Kerala: Research Signpost; 2007:69-80.

12. Tsiklauri N, Viatchenko-Karpinsky V, Voitenko N, Tsagareli MG. Non-opioid tolerance in juvenile and adult rats. Eur J Pharmacol. 2010;629(1-3):68-72.

13. Gurtskaia G, Tsiklauri N, Nozadze I, Nebieridze M, Tsagareli MG. Antinociceptive tolerance to NSAIDs microinjected into dorsal hippocampus. BMC Pharmacol Toxicol. 2014;15:10.

14. Tsagareli MG, Nozadze I, Tsiklauri N, Gurtskaia G. Tolerance to nonopioid analgesics is opioid sensitive in the nucleus raphe magnus. Front Neurosci. 2011;5:92.

15. Tsagareli MG, Tsiklauri N, Gurtskaia G, Nozadze I, Abzianidze E. The central nucleus of amygdala is involved in tolerance to the antinociceptive effect of NSAIDs. Health. 2010;2(1):64-68.

16. Vanegas H, Vazquez E, Tortorici V. NSAIDs, opioids, cannabinoids and the control of pain by the central nervous system. Pharmaceuticals. 2010;3:1335-1347.

17. Zimmermann M. Ethical guidelines for investigations of experimental pain in conscious animals. Pain. 1983;16(2):109-110.

18. Paxinos G, Watson C. The Rat Brain in Stereotaxic Coordinates. Compact third edition. San Diego, CA: Academic Press; 1997.

19. Tortorici V, Vanegas H. Opioid tolerance induced by metamizol (dipyrone) microinjections into the periaqueductal gray of rats. Eur $J$ Neurosci. 2000;12(11):4074-4080.

20. Tsiklauri N, Nozadze I, Firkulashvili N, et al. Cellular mechanisms of antinociceptive tolerance to non-steroidal anti-inflammatory drugs. In: Systemic, Cellular and Molecular Mechanisms of Physiological Functions and Their Disorders. Chapter 26. New York, NY: Nova Biomedical; 2016:339-362. 
21. Telleria-Diaz A, Schmidt M, Kreusch S, et al. Spinal antinociceptive effects of cyclooxygenase inhibition during inflammation: involvement of prostaglandins and endocannabinoids. Pain. 2010;148(1): 26-35.

22. Tortorici V, Nogueira L, Salas R, Vanegas H. Involvement of local cholecystokinin in the tolerance induced by morphine microinjections into the periaqueductal gray of rats. Pain. 2003;102(1-2):9-16.

23. Heinricher MM, Ingram SL. The brainstem and nociceptive modulation. In: Basbaum AI, Bushnell MC, editors. Science of Pain. San Diego, CA: Elsevier; 2009:593-626.

24. Heinricher MM, Fields HL. Central nervous system mechanisms of pain modulation. In: McMahon SB, Koltzenburg M, Tracey I, Turk DC, editors. Wall \& Melzack's Textbook of Pain. Philadelphia: Elsevier; 2013:129-142.
25. Tsiklauri N, Nozadze I, Gurtskaia G, Tsagareli MG. Antinociceptive tolerance to NSAIDs in the rat formalin test is mediated by the opioid mechanism. Pharmacol Reports. 2017;69(1):168-175.

26. Vazquez E, Hernandez N, Escobar W, Vanegas H. Antinociception induced by intravenous dipyrone (metamizol) upon dorsal horn neurons: involvement of endogenous opioids at the periaqueductal gray matter, the nucleus raphe magnus, and the spinal cord in rats. Brain Res. 2005;1048(1-2):211-217.

27. Hernández-Delgadillo GP, Cruz SL. Endogenous opioids are involved in morphine and dipyrone analgesic potentiation in the tail flick test in rats. Eur J Pharmacol. 2006;546(1):54-59.

28. Vazquez E, Escobar W, Ramirez C, Vanegas H. A non-opioid analgesic acts upon the PAG-RVM axis to reverse inflammatory hyperalgesia. Eur J Neurosci. 2007;25(2):471-479.

\section{Publish your work in this journal}

The Journal of Pain Research is an international, peer reviewed, open access, online journal that welcomes laboratory and clinical findings in the fields of pain research and the prevention and management of pain. Original research, reviews, symposium reports, hypothesis formation and commentaries are all considered for publication.

\section{Dovepress}

The manuscript management system is completely online and includes a very quick and fair peer-review system, which is all easy to use. Visit http://www.dovepress.com/testimonials.php to read real quotes from published authors. 